\title{
On Digital Line and Operations
}

\author{
Abd El-Monem M. Kozae ${ }^{1}$ and El-Sayed A. Abo-Tabl ${ }^{2}$ \\ ${ }^{1}$ Mathematics Department, Faculty of Science, Tanta University, Tanta, Egypt \\ ${ }^{2}$ Mathematics Department, Faculty of Science, Assiut University, Assiut, Egypt \\ 2 Mathematics Department, Faculty of Science and Arts, Qassim University, Muthnab, KSA \\ ${ }^{1}$ akozae55@yahoo.com, ${ }^{2}$ abotabl@yahoo.com
}

\begin{abstract}
The topological structure is a more general mathematical model that helps in solving many problems in digital topology and its applications. In this paper, we introduce and study some general forms of separation properties based on operations. The notions of generalized closed set and $T_{1 / 2}$-spaces are further investigated using operation approaches. As applications, it is shown that the Khalimsky line (digital line) is a typical example of $\mu_{r, s}-T_{1 / 2}$-spaces, where $\mu_{r}=i n t$ and $\mu_{s}=i n t-c l$.
\end{abstract}

Keywords: Operations, $T_{1 / 2}$-spaces, digital line, topological space.

2010 MSC No: 54A05; $11 B 05$

\section{Introduction}

General topology has applications in the theory of image processing by exhibiting algorithms which applies to the current knowledge of digital topology (i.e., the study of the geometric and topological properties of digital images). Problems that might arise are, for example, finding connected components, set boundaries or any other operations which are needed in image processing. The well-known digital Jordan curve theorem is proved by using topological approach $([17,19,29])$. The theorem is important in the theory of computer graphics.

Topologically speaking, the computer and the television screen look like a rectangular portion of $R^{2}$ granted. They are greatly magnified as revealing themselves as a rectangular or hexagonal array of dots. In the process of digitizing a movie, time is discretized, resulting in a finite seeming rectangular solid in $R^{3}$.

These situations are often represented by subspaces and quotients of locally finite topological spaces, so their study is important. The basic building block of the digital $n$-space is the digital line or the so called Khalimsky line $([17,18,20,22])$. This is the set of the integers, $Z$, equipped with the topology $K$, generated by $S_{K}=$ $\{\{2 n-1,2 n, 2 n+1\}: n \in Z\}$ as a subbase. Thus a set $U$ is open in $K$ if and only if whenever $x \in U$ is an even integer, then $x-1, x+1 \in U$. Nowadays, this topology, called the Khalimsky topology, is one of the most important concepts of the digital topology. It has been studied and used by many authors, e.g., ([2-11, 15, 17-20, 22, 24-29]).

The digital line, the digital plane, the three-dimensional digital space are of great importance in the study of applications of point-set topology to computer graphics [17]. Papers [12, 14, 20, 23, 28], are topological approaches of digital spaces, that is "topological digital topology".

The concept of operations on the power set was introduced and investigated by Abd El-Monsef et al [1]. In this paper, we investigate "operation-generalized closed set", a characterization of "operation- $T_{1 / 2}$-space" and onepoint compactification of some "operation- $T_{1 / 2}$-space". As application, we show that the Khalimsky line is a typical example of $\mu_{r, s}-T_{1 / 2}$-spaces, where $\mu_{r}=i n t$ and $\mu_{s}=i n t-c l$. 


\section{Preliminaries}

In the present article we review Some definitions and Properties from [1].

Definition 2.1 A function $\mu: P(X) \rightarrow P(X)$ is said to be an operation on $P(X)$ if $\operatorname{int}(A) \subset A^{\mu}$ for every $A \in P(X)$, where $\mu(A)=A^{\mu}$.

The operation $\mu, \mu^{\prime}$ and $\mu^{\prime \prime}$ defined by $\mu(V)=\operatorname{int}(V), \mu^{\prime}(V)=\operatorname{int}(\operatorname{cl}(V))$ and $\mu^{\prime \prime}(V)=\operatorname{int}(\operatorname{cl}(\operatorname{int}(V)))$ for all $V \in P(X)$ are examples of operations on $P(X)$. The operation $\mu\left(\right.$ resp. $\left.\mu^{\prime}, \mu^{\prime \prime}\right)$ above is called the "interior operation" (resp. "interior-closure operation", "interior-closure-interior operation").

Definition 2.2 An operation $\mu: P(X) \rightarrow P(X)$ is said to be regular with respect to $\tau \subset P(X)$ if for every $U, V \in \tau$ and $x \in U \cup V$ where $x \in X$, there exists $W \in \tau$ with $x \in X$ such that $W^{\mu} \subset U^{\mu} \cap V^{\mu}$.

Definition 2.3 An operation $\mu: P(X) \rightarrow P(X)$ is said to be monotone if $A \subset B$ implies $A^{\mu} \subset B^{\mu}$. The operation $\mu, \mu^{\prime}$ and $\mu^{\prime \prime}$ above are monotone.

Definition 2.4 Let $(X, \tau)$ be a topological space and $A \in P(X)$, then $A$ is said to be $\mu_{r}-$ open if $A \subset A^{\mu_{r}}$. $A$ is said to be $\mu_{r}$-closed if $X-A$ is $\mu_{r}$-open.

Definition 2.5 $A$ is said to be regular $\mu_{r}$-open if $A=A^{\mu_{r}}$. $A$ is said to be regular $\mu_{r}-$ closed if $X-A$ is regular $\mu_{r}$-open.

Definition 2.6 A point $x \in A$ is in the $\mu_{r, s}$-interior of $A$, written $x \in \mu_{r, s}-i n t(A)$, if there exists $\mu_{r}-$ open $U$ contains $x$ such that $U^{\mu_{r}} \subset A$. A subset $A$ of $X$ is $\mu_{r, s}$-open if $A=\mu_{r, s}-\operatorname{int}(A)$.

Definition 2.7 A point $x \in A$ is in the $\mu_{r, s}$-closure of $A$, written $x \in \mu_{r, s}-\operatorname{cl}(A)$, if for every $\mu_{r}-$ open $U$ contains $x$, then $U^{\mu_{r}} \cap A \neq \phi$. A subset $A$ of $X$ is $\mu_{r, s}-\operatorname{closed}$ if $A=\mu_{r, s}-\operatorname{cl}(A)$.

Theorem 2.1 Let $\mu_{r}, \mu_{s}: P(X) \rightarrow P(X)$ be two operations on $P(X)$ and $A, B \in P(X)$, then

(i) $A$ is $\mu_{r, s}$-closed if and only if $X-A$ is $\mu_{r, s}-$ open.

(ii) If $\mu_{r}$ is monotone and $A_{i}$ is $\mu_{r}$-open for every $i \in I$, then $\cup_{i \in I} A_{i}$ is $\mu_{r}$-open.

(iii) If $\mu_{r}$ is monotone, then $A$ is $\mu_{r}$-closed if and only if for every $x \notin A$ there exists $\mu_{r}$-open set $U$ such that $U \cap A=\phi$.

(iv) If $\mu_{r}$ is monotone, then $A$ is $\mu_{r}$-open if and only if for every $x \in A$ there exists $\mu_{r}$-open set $U$ such that $U \subset A$.

(v) If $\mu_{s}$ is regular with respect to the family of all $\mu_{r}$-open sets in $X$, then $\mu_{r, s}-\operatorname{cl}(A \cup B)=\mu_{r, s}-\operatorname{cl}(A) \cup \mu_{r, s}-\operatorname{cl}(B)$.

Definition 2.8 [16] Let $(X, \tau)$ be any topological space. We shall define the one-point compactification of $(X, \tau)$ which we denote by $\left(X^{*}, \tau^{*}\right)$. Here:

(i) $X^{*}=X \cup\{p\}$, where $p$, is distinct from every other point in $X$,

(ii) $\tau^{*}$ consists of the following sets:

(a) each member of the topology $\tau$ on $X$,

(b) the complement in $X^{*}$ of any closed and compact subset of $X$.

Since $X$ is open in $\left(X^{*}, \tau^{*}\right)$ and $\tau_{X}^{*}=\tau$ holds, we have the following properties for a subset $A$ of $X,[22]$

$$
\begin{gathered}
\tau-\operatorname{int}(A)=\tau_{X}^{*}-\operatorname{int}(A)=\left(\tau^{*}-\operatorname{int}(A)\right) \cap X ; \\
\tau-\operatorname{cl}(A)=\tau_{X}^{*}-\operatorname{cl}(A)=\left(\tau^{*}-\operatorname{cl}(A)\right) \cap X ; \\
\left.((\tau-i n t)-(\tau-c l))(A)=\left(\left(\tau^{*}-i n t\right)-\left(\tau^{*}-c l\right)\right)(A)\right) \cap X ;
\end{gathered}
$$

Definition 2.9 [23] A topological space $(X, \tau)$ has a (*-property) if for every $x \in X$ there exists an open neighborhood $U$ of the point $x$ such that $\operatorname{cl}(U)$ is a compact subspace of $(X, \tau)$. 


\section{$3 \quad \mu_{r, s}-$ generalized closed sets and $\mu_{r, s}-T_{1 / 2}-$ spaces}

In this section we introduce $\mu_{r, s}$-generalized closed sets (short, $\mu_{r, s}-g$-closed sets) and $\mu_{r, s}-T_{1 / 2}-$ spaces and obtain their characterizations.

Definition 3.1 A subset $A$ of $X$ is said to be $\mu_{r, s}-g$-closed set if $\mu_{r, s}-\operatorname{cl}(A) \subset U$ whenever $A \subset U$ and $U$ is $\mu_{r, s}$-open.

Proposition 3.1 Every $\mu_{r, s-}-$ closed set is $\mu_{r, s}-g$-closed.

Proof. Let $A$ be a $\mu_{r, s}$-closed subset of $X$ contained in a $\mu_{r, s}$ open set $U$, i.e., $A \subset U$, then $\mu_{r, s}-c l(A) \subset U$, therefore $A$ is a $\mu_{r, s}-g$-closed set.

Here, we give a counter example to prove that the converse of the previous proposition is not true in general.

Example 3.1 Let $\tau=\{\phi,\{a\},\{b\},\{a, c\},\{a, b\}, X\}$ be a topology define on $X=\{a, b, c\}$. Let $\mu_{r}, \mu_{s}: P(X) \rightarrow P(X)$ be two operations on $P(X)$ defined as follows: for every $A \in P(X), A^{\mu_{r}}=\operatorname{int}(A)$ and $A^{\mu_{s}}=\operatorname{cl}(A)$. Since $\mu_{r, s}-c l(\{b, c\})=X$, then $\{b, c\}$ is not $\mu_{r, s}$-closed. However, $\{b, c\}$ is $\mu_{r, s}-g$-closed in $(X, \tau)$ because $X$ is the only $\mu_{r, s}$-open set containing $\{b, c\}$.

Proposition 3.2 Suppose that $\mu_{s}: P(X) \rightarrow P(X)$ is a regular operation on $P(X)$. If $A$ and $B$ are two $\mu_{r, s}-g-$ closed sets, then $A \cup B$ is $\mu_{r, s}-g$-closed too.

Proof. Let $U$ be $\mu_{r, s}$-open and $A \cup B \subset U$. Since $A$ and $B$ are two $\mu_{r, s}-g$-closed sets, then $\mu_{r, s}-c l(A) \subset U$ and $\mu_{r, s}-\operatorname{cl}(B) \subset U$. Hence, $\mu_{r, s}-\operatorname{cl}(A) \cup \mu_{r, s}-\operatorname{cl}(B) \subset U$. Since $\mu_{s}$ is regular, thus $\mu_{r, s}-\operatorname{cl}(A \cup B) \subset U$, i.e., $A \cup B$ is $\mu_{r, s}-g$-closed.

Proposition 3.3 If a subset $A$ of a topological space $(X, \tau)$ is $\mu_{r, s}-g-\operatorname{closed}$, then $\mu_{r, s}-\operatorname{cl}(A)-A$ does not contain any nonempty $\mu_{r, s}-$ closed set.

Proof. Let $F$ be a $\mu_{r, s}$-closed set contained in $\mu_{r, s}-\operatorname{cl}(A)-A$. Then, $X-F$ is a $\mu_{r, s}-$ open set containing $A$, it follows from the assumption that $\mu_{r, s}-\operatorname{cl}(A) \subset X-F$. Hence, $F \subset\left(\mu_{r, s}-\operatorname{cl}(A)-A\right) \cap\left(X-\mu_{r, s}-\operatorname{cl}(A)\right) \subset \phi$ and so $F=\phi$.

Proposition 3.4 For each $x \in X,\{x\}$ is either $\mu_{r, s}$-closed or its complement $X-\{x\}$ is $\mu_{r, s}-g$-closed.

Proof. Suppose that $\{x\}$ is not $\mu_{r, s}$-closed. Then, its complement $X-\{x\}$ is not $\mu_{r, s}-$ open. $X$ is the only $\mu_{r, s}$-open set containing $X-\{x\}$ and $\mu_{r, s}-\operatorname{cl}(X-\{x\}) \subset X$, thus $X-\{x\}$ is $\mu_{r, s}-g$-closed.

Definition 3.2 A topological space $(X, \tau)$ is said to be $\mu_{r, s}-T_{1 / 2}$-space if every $\mu_{r, s}-g-$ closed set is $\mu_{r, s}-$ closed.

We shall illustrate the above definition by the following example.

Example 3.2 The discrete space $P(X)$ is $\mu_{r, s}-T_{1 / 2}-$ space whenever $\mu_{r}=i n t$ and $\mu_{s}=i n t-c l$. But the topological space in Example 3.1 is not $\mu_{r, s}-T_{1 / 2}-$ space, because the set $\{b, c\}$ is $\mu_{r, s}-g-$ closed but it is not $\mu_{r, s}-$ closed.

Theorem 3.1 For the topological space $(X, \tau)$ and the operations $\mu_{r}, \mu_{s}: P(X) \rightarrow P(X)$ on $P(X)$, the following properties are equivalent.

(i) $(X, \tau)$ is $\mu_{r, s}-T_{1 / 2}$,

(ii) Every singleton $\{x\}$ is $\mu_{r, s}-$ open or $\mu_{r, s}-$ closed.

Proof. $(i \rightarrow i i)$, Let $(X, \tau)$ be a $\mu_{r, s}-T_{1 / 2}-$ space. Suppose that, $\{x\}$ is not $\mu_{r, s}-$ closed for some $x \in X$. Then, from Proposition 3.4, we have $X-\{x\}$ is a $\mu_{r, s}-g$-closed set. Therefore, $X-\{x\}$ is a $\mu_{r, s}-$ closed set and so $\{x\}$ is $\mu_{r, s}$-open.

$(i i \rightarrow i)$, Let $A$ be a $\mu_{r, s}-g-\operatorname{closed}$ set. Then, we claim that $\mu_{r, s}-\operatorname{cl}(A)=A$ holds. Let $x \in \mu_{r, s}-\operatorname{cl}(A)$. By the 
assumption $\{x\}$ is $\mu_{r, s}$-open or $\mu_{r, s}$-closed. Hence, there are two cases.

Case 1. suppose that $\{x\}$ is $\mu_{r, s}$-open, by definition, there is $\mu_{r}$-open set $U$ containing $x$ such that

$$
U_{s}^{\mu} \subset\{x\}
$$

Since $x \in \mu_{r, s}-c l(A)$, then for every $\mu_{r}$-open set $U$ containing $x$, we get

$$
U_{s}^{\mu} \cap A \neq \phi
$$

From (1) and (2) we have $U_{s}^{\mu}=\{x\}$ and so $x \in A$.

Case 2. Suppose that $\{x\}$ is $\mu_{r, s}$-closed. Then, from Proposition 3.3 we get $\mu_{r, s}-\operatorname{cl}(A)-A$ does not contain $\{x\}$. And hence, $x \in A$.

Therefore, $\mu_{r, s}-\operatorname{cl}(A) \subset A$, and so $A$ is $\mu_{r, s}$-closed. Thus, $(X, \tau)$ is a $\mu_{r, s}-T_{1 / 2}-$ space.

In [13] Dontchev and Ganster defined $\delta$-generalized closed sets and investigated the class of $T_{3 / 4}-$ spaces, which is properly placed between the class of $T_{1}$-spaces and $T_{1 / 2}-$ spaces.

Definition 3.3 [13] A topological space $(X, \tau)$ is $T_{3 / 4}$ if and only if every singleton $\{x\}$ is $\delta$-open or closed.

Definition $3.4[12]$ A topological space $(X, \tau)$ is almost weakly Hausdorff if and only if every singleton $\{x\}$ is $\delta$-open or $\delta$-closed.

In [1] it is shown that, in $(X, \tau)$, a subset $A$ is $\mu_{r, s}-$ closed where $\mu_{r}=i n t$ and $\mu_{s}=i n t-c l$ if and only if $A$ is $\delta$-closed, i.e., a subset $A$ is $\mu_{r, s}$-open if and only if $A$ is $\delta$-open.

Corollary 3.1 (i) A topological space is $\mu_{r, s}-T_{1 / 2}$ where $\mu_{r}=$ int and $\mu_{s}=$ int $-c l$ if and only if it is almost weakly Hausdorff.

(ii) Every $\mu_{r, s}-T_{1 / 2}-$ space where $\mu_{r}=i n t$ and $\mu_{s}=i n t-c l$ is a $T_{3 / 4}-$ space.

It was shown, in [12], that the Khalimsky line $(Z, K)$ is almost weakly Hausdorff space, then from Corollary 3.1 we present the following lemma.

Lemma 3.1 The Khalimsky line $(Z, K)$ is a typical example of $\mu_{r, s}-T_{1 / 2}$ where $\mu_{r}=i n t$ and $\mu_{s}=i n t-c l$, it is not $T_{1}$.

Remark 3.1 We note that every singleton set $\{2 n+1\}, n \in Z$ is $\mu_{r, s}$-open and every singleton set $\{2 n\}, n \in Z$ is $\mu_{r, s}-$ closed in $(Z, K)$.

Remark 3.2 The $\mu_{r, s}-T_{1 / 2}$-axioms, where $\mu_{r}=$ int and $\mu_{s}=i n t-c l$, is independent of the $T_{1}-$ separation axiom. In fact, the digital line $(Z, K)$ is not $T_{1}$; it is $\mu_{r, s}-T_{1 / 2}$. The real line with the cofinite topology is an example of a $T_{1}$-space, which is not $\mu_{r, s}-T_{1 / 2}$.

Lemma 3.2 A subset $U$ is $\mu_{r}$-open, where $\mu_{r}=i n t$, if and only if $U$ is open.

Proof. Assume that $U$ is $\mu_{r}$-open, where $\mu_{r}=i n t$, then $U \subset \operatorname{int}(U)$, thus $U=\operatorname{int}(U)$ and so $U$ is open. Conversely, suppose $U$ is open, hence $U=\operatorname{int}(U)$, therefore $U \subset \operatorname{int}(U)$, i.e., $U$ is $\mu_{r}$-open, where $\mu_{r}=i n t$.

From Theorem 4.9 [23. 0], Corollary 3.1 and Lemma 3.2 we have the following theorem.

Theorem 3.2 If $(X, \tau)$ has $\left(*-\right.$ property) and $\tau-\mu_{r, s}-T_{1 / 2}$, then the one point compactification $\left(X^{*}, \tau^{*}\right)$ is $\tau^{*}-\mu_{r, s}-T_{1 / 2}$, where $\mu_{r}=$ int and $\mu_{s}=$ int $-c l$.

proof. For a point $x \neq p$, there exists an open neighborhood of $x$, say $U(x)$, such that $c l(U(x))$ is a compact subspace of $(X, \tau)$. Let $S(x)=(X-c l(U(x))) \cup\{p\}$. Then we note that the following properties hold:

$(* *) x \in U(x), U(x) \in \tau$ and $\tau^{*}-\operatorname{cl}(U(x)) \cap\{p\}=\phi$, especially, $\tau^{*}-\operatorname{cl}(\{x\}) \cap\{p\}=\phi$,

$(* * *) p \in S(x), S(x) \in \tau^{*}$ and $\tau^{*}-\operatorname{cl}(S(x)) \subset X-\{x\}$. 
Let $\{x\}$ be a singleton of $X^{*}$. We want to prove that, in $\left(X^{*}, \tau^{*}\right)$, if $x \neq p$, then $\{x\}$ is $\tau^{*}-\mu_{r, s}-$ open or $\tau^{*}-\mu_{r, s}$-closed and if $x=p$, then $\{p\}$ is $\tau^{*}-\mu_{r, s}-$ closed, where $\mu_{r}=$ int and $\mu_{s}=$ int $-c l$. Using assumption that $(X, \tau)$ is $\mu_{r, s}-T_{1 / 2}$, from Theorem 3.1 and definition 2.6, we have three cases. We abbreviate $\tau-\operatorname{int}($.$) and$ $\tau-\operatorname{cl}($.$) by \operatorname{int}($.$) and \operatorname{cl}($.$) , respectively, in the proof below.$

Case 1. If $x \neq p$ and $\{x\}$ is $\mu_{r, s}$-open in $(X, \tau)$. In this case, $\{x\}$ is a unique nonempty $\mu_{r}$-open set contained in $\{x\}, \operatorname{int}(\operatorname{cl}(\{x\}))=\{x\}$ holds in $(X, \tau)$ and also $\{x\}$ is $\tau^{*}-\mu_{r}$-open. By $(* *)$ it is shown that

$$
\begin{aligned}
\tau^{*}-\operatorname{cl}(\{x\}) & =\tau^{*}-\operatorname{cl}(\{x\}) \cap X^{*} \\
& =\tau^{*}-\operatorname{cl}(\{x\}) \cap(X \cup\{p\}) \\
& =\left(\tau^{*}-\operatorname{cl}(\{x\}) \cap X\right) \cup\left(\tau^{*}-\operatorname{cl}(\{x\}) \cap\{p\}\right) \\
& =\left(\tau^{*}-\operatorname{cl}(\{x\}) \cap X\right) \cup \phi \\
& =\tau_{X}^{*}-\operatorname{cl}(\{x\} \\
& =\tau-\operatorname{cl}(\{x\}) \\
& =\operatorname{cl}(\{x\})
\end{aligned}
$$

and so

$$
\begin{aligned}
\tau^{*}-\operatorname{int}\left(\tau^{*}-\operatorname{cl}(\{x\})\right) & =\tau^{*}-\operatorname{int}(\operatorname{cl}(\{x\})) \\
& =\tau^{*}-\operatorname{int}(\operatorname{cl}(\{x\})) \cap X \\
& =\tau_{X}^{*}-\operatorname{int}(\operatorname{cl}(\{x\})) \\
& =\tau-\operatorname{int}(\tau-\operatorname{cl}(\{x\})) \\
& =\operatorname{int}(\operatorname{cl}(\{x\})) \\
& =\{x\} .
\end{aligned}
$$

Thus we show that $\{x\}$ is $\mu_{r, s}$-open in $\left(X^{*}, \tau^{*}\right)$.

Case 2. If $x \neq p$ and $\{x\}$ is $\mu_{r, s}-$ closed in $(X, \tau)$. Let $y \in X^{*}-\{x\}$. Firstly, we suppose that $y \neq p$, then $y \in X-\{x\}$ and there exists a $\mu_{r}$-open set $V$ such that $\operatorname{int}(\operatorname{cl}(V)) \subset X-\{x\}$ and $y \in V$. Then,

$$
\begin{aligned}
\tau^{*}-\operatorname{int}\left(\tau^{*}-\operatorname{cl}(V)\right) & =\left(\tau^{*}-\operatorname{int}\left(\tau^{*}-\operatorname{cl}(V)\right)\right) \cap X^{*} \\
& \subset\left(\tau^{*}-\operatorname{int}(\tau-\operatorname{cl}(V))\right) \cap X^{*} \\
& \subset\left(\left(\tau^{*}-\operatorname{int}(\tau-\operatorname{cl}(V))\right) \cap X\right) \cup\left(\left(\tau^{*}-\operatorname{int}(\tau-\operatorname{cl}(V))\right) \cap\{p\}\right) \\
& \subset\left(\tau_{X}^{*}-\operatorname{int}(\tau-\operatorname{cl}(V))\right) \cup((\tau-\operatorname{cl}(V)) \cap\{p\}) \\
& \subset \operatorname{int}(\operatorname{cl}(V)) \cup \phi \\
& \subset \operatorname{int}(\operatorname{cl}(V))
\end{aligned}
$$

and so we have $\tau^{*}-\operatorname{int}\left(\tau^{*}-\operatorname{cl}(V)\right) \subset X^{*}-\{x\}$.

Next, we suppose that $y=p$. Since $x \neq p$, by using $(* * *)$ for $x$, then there exists a subset $S(x) \in \tau^{*}$ which is $\mu_{r}$-open and $y=p \in S(x)$ and $\tau^{*}-\operatorname{int}\left(\tau^{*}-\operatorname{cl}(S(x))\right) \subset X^{*}-\{x\}$. Therefore, in this case, $X^{*}-\{x\}$ is $\tau^{*}-\mu_{r, s}-$ open and hence $\{x\}$ is $\tau^{*}-\mu_{r, s}-$ closed in $\left.X^{*}, \tau^{*}\right)$.

Case 3. If $x=p$. Let $y \in X^{*}-\{p\}$. Since $y \neq p$, by $(* *)$ for $y$ above, there exists a subset $U(y) \in \tau$ containing $y$ such that $\left.\tau^{*}-c l(U(y))\right) \subset X^{*}-\{p\}$, where $U$ is $\mu_{r}$-open. Therefore, we have

$$
\tau^{*}-\operatorname{int}\left(\tau^{*}-\operatorname{cl}(U)\right) \subset \tau^{*}-\operatorname{cl}(U) \subset X^{*}-\{p\}
$$

and hence $X^{*}-\{p\}$ is $\tau^{*}-\mu_{r, s}$-open, i.e., $\{x\}$ is $\tau^{*}-\mu_{r, s}-\operatorname{closed}$ in $\left.X^{*}, \tau^{*}\right)$.

From Theorem 3.2 and Lemma 3.1 we present the following lemma.

Lemma 3.3 The one point compactification $\left(Z \cup\{p\}, K^{*}\right)$ of the digital line $(Z, K)$ is one of typical examples of $\mu_{r, s}-T_{1 / 2}-$ space, where $p$ is a point not in $Z$, where $\mu_{r}=i n t$ and $\mu_{s}=i n t-c l$.

Remark 3.3 The singleton $\{p\}$ and every singleton $\{2 n\}, n \in Z$, are $\mu_{r, s}-$ closed and every singleton $\{2 n+1\}$, $n \in Z$, is $\mu_{r, s}$-open, where $\mu_{r}=i n t$ and $\mu_{s}=i n t-c l$. 


\section{Conclusion}

In short topology is a branch of mathematics, whose concepts are not only fundamental to all branches of mathematics, but also in real life applications.

Image plays important role in real life. In the past the process of image analysis took place via various mathematical models with an acceptable amount of error. Digital topology is a new accurate approach.

The results of this work help in constructing digital images in product spaces, and this in turn can help in obtaining more precise image in fields such as medicine and geography.

\section{Acknowledgements}

The author would like to thank the anonymous referees and the Editor-in-Chief, for their valuable suggestions in improving this paper.

\section{References}

[1] M. E. Abd El-Monsef, F. M. Zeyada A. S. Mashhour, Operations on the power set $P(X)$ of a topological space $(X, \tau)$, Colloquium on topology, Eger-Hungary, August 9-13 (1983).

[2] Y. Bai, X. Han J. L. Prince, Digital topology on adaptive Octree grids, Journal of mathematical imaging and vision, (34) (2009) 165-184. https://doi.org/10.1007/s10851-009-0140-7

[3] Li. Boxer, Li Remarks on digitally continuous multivalued functions, J. Adv. Math. 9(1) (2014), $1755-1762$.

[4] Li. Boxer, Digital shy maps, Appl. Gen. Topol. 18(1) (2017), 143-152.https://doi.org/10.4995/agt.2017.6663

[5] Li. Boxer, Generalized normal product adjacency in digital topology, Applied General Topology 18 (2) (2017), 401-427. https://doi.org/10.4995/agt.2017.7798

[6] Li. Boxer, Alternate product adjacencies in digital topology, Applied General Topology, 19(1) (2018), 21-53. https://doi.org/10.4995/agt.2018.7146

[7] Li. Boxer, Shy maps in topology: Topology and its Applications, 242(2018),59-65. https://doi.org/10.1016/j.topol.2018.04.017

[8] Li. Boxer P.C. Staecker, Connectivity preserving multivalued functions in digital topology, J. Math. Imaging Vis. 55(3) (2016), 370-377, https://doi .org /10 .1007/s10851 -015 -0625 -5.

[9] Li. Boxer P.C. Staecker, Remarks on Pointed Digital Homotopy, Topology Proceedings, 51 (2018), 19-37

[10] Li. Boxer P.C. Staecker, Remarks on Fixed Point Assertions in Digital Topology, Applied General Topology, to appear.

[11] U.P. Dolhare V.V. Nalawade, Fixed point theorems in digital images and applications to fractal image compression, Asian Journal of Mathematics and Computer Research, 25 (1) (2018), 18-37.

[12] J. Dontchev, I. Arokiarani K. Balachandran, On generalized $\delta$ - closed sets and almost weakly Hausdorff spaces, Questions and Answers in General Topology, 18(1) (2000), 1-14.

[13] J. Dontchev M. Ganster, On $\delta$-generalized closed sets and $T_{3 / 4}-$ spaces, Mem. Fac. Sci. Kochi Univ. Math. 17(1996), 15-31.

[14] J. Dontchev H. Maki, Groups of $\theta$-generalized homeomorphisms and the digital line, Topology and its Applications, 95(1999) 113-128.https://doi.org/10.1016/s0166-8641(98)00004-2

[15] D. Jain, Common fixed point theorem for intimate mappings in digital metric spaces, International Journal of Mathematics Trends and Technology, 56 (2) (2018), 91-94.https://doi.org/10.14445/22315373/ijmtt-v56p511

[16] J. L. Kelley, General Topology, (PWN, New York, 1955). 
[17] E. D. Khalimsky, R. Kopperman P. R. Meyer, Computer graphics and connected topologies on finite ordered sets, topology and its application, 36(1990) 1-17.https://doi.org/10.1016/0166-8641(90)90031-v

[18] E. D. Khalimsky, R. Kopperman P. R. Meyer, Boundaries in digital planes, Journal of Applied Mathematics and Stochastic Analysis, 3 (1990) 27-55.https://doi.org/10.1155/s1048953390000041

[19] O. C. Kiselman, Digital Jordan Curve theorems, Discrete Geometry for Computer Imagery, (DGCI)(2000) 46-56. https://doi.org/10.1007/3-540-44438-65

[20] T. Y. Kong, R. Kopperman P. R. Meyer, A topological approach to digital topology, Am, Math. Monthly, 98 (1991) 901-917.https://doi.org/10.1080/00029890.1991.12000810

[21] T. Y. Kong A. Rosenfeld, A Digital topology: Introduction and survey, Computer Vision and Image Processing, 48(1989) 357-393.https://doi.org/10.1016/0734-189x(89)90147-3

[22] R. Kopperman, P. R. Meyer R. G. Wilson, A Jordan surface theorem for three-dimensional digital spaces, Discrete and computational Geometry, 6 (1991) 155-162.https://doi.org/10.1007/bf02574681

[23] H. Maki, H. Ogata, K. Balachandran, P. Sundaram R. Devi, The digital line and operation approaches of $T_{1 / 2}$-spaces, Scientice Mathematicae, 3(3)(2000), 345-352.

[24] E. Melin, Digital surfaces and boundaries in Khalimsky spaces, Journal of mathematical imaging and vision, (28) (2007) 169-177.https://doi.org/10.1007/s10851-007-0006-9

[25] E. Melin, Digital Khalimsky manifolds, J. Math. Imaging Vis. $33 \quad$ (2009) 267280.https://doi.org/10.1007/s10851-008-0114-1

[26] L. N. Mishra, K. Jyoti, A. Rani Vandana, Fixed point theorems with digital contractions image processing, Nonlinear Science Letters A, 9 (2)(2018), 104-115.

[27] J. Slapal, A Jordan curve theorem with respect to certain closure operations on the digital plane, Electronic Notes in Theoretical Computer Science, 46(2001) 1-20.https://doi.org/10.1016/s1571-0661(04)80978-2

[28] J. Slapal, Closure operations for digital topology, Theoretical Computer Science, 305(2003)457471.https://doi.org/10.1016/s0304-3975(02)00708-9

[29] J. Slapal, Digital Jordan curves, topology and its application, 135(2006) 32553264.https://doi.org/10.1016/j.topol.2005.10.011 